\title{
Costs and Benefits of Behavioral Specialization
}

\author{
Arne Brutschy ${ }^{1}$, Nam-Luc Tran ${ }^{1}$, Nadir Baiboun ${ }^{1,2}$, Marco Frison ${ }^{1,3}$, \\ Giovanni Pini ${ }^{1}$, Andrea Roli ${ }^{3,1}$, Marco Dorigo ${ }^{1}$, and Mauro Birattari ${ }^{1}$ \\ 1 IRIDIA, CoDE, Université Libre de Bruxelles, Brussels, Belgium \\ 2 ECAM, Institut Supérieur Industriel, Brussels, Belgium \\ 3 DEIS-Cesena, Alma Mater Studiorum Università di Bologna, Cesena, Italy \\ arne.brutschy@ulb.ac.be
}

\begin{abstract}
In this work, we study behavioral specialization in a swarm of autonomous robots. In the studied swarm, a robot working repeatedly on the same type of task improves in task performance due to learning. Robots may exploit this positive effect of learning by selecting with higher probability the tasks on which they have improved their performance. However, even though the exploitation of such performanceimproving effects is clearly a benefit, specialization also entails certain costs. Using a task allocation strategy that allows the robots to behaviorally specialize, we study the trade-off between costs and benefits in simulation experiments. Additionally, we give a perspective on the impact of this trade-off in systems that use specialization.
\end{abstract}

Keywords: specialization, task allocation, swarm robotics, swarm intelligence, self-organization, division of labor.

\section{Introduction}

Division of labor is a concept that is common in the organization of large groups of individuals such as humans or social insects [115]. In division of labor, "(a) each worker specializes on a subset of the complete repertoire of tasks performed by the colony, and (b) this subset varies across individual workers in the colony" [1. A common way to obtain division of labor is to let individuals adapt their behavior to focus on a subset of the tasks available - this is called behavioral specialization 13 . Behavioral specialization is known to increase the overall performance of an individual because of many different reasons. One of the most important of these reasons is learning: an individual grows more efficient in performing a task by repeating it multiple times 15. A specialized individual can exploit this increased efficiency by behaviorally focusing on the tasks it is good at.

In this work, we study the costs and benefits of behavioral specialization in a swarm of autonomous robots. In the studied system, robots have to perform different types of tasks which are independent of each other. The robots can improve their performance on a task by repeatedly working on it (i.e., they learn). In order to maximize this performance improvement, robots should focus on a single task - first to learn this task to the full extent, and second to fully exploit the resulting performance improvement. We propose a simple self-organized strategy 
for task allocation that allows the robots to behaviorally specialize on a specific task, thereby maximizing the performance improvement available through learning.

However, even though the exploitation of such a performance improvement is clearly a benefit, specialization also entails certain costs [15. An example for such a cost is the time a specialized robot spends searching for a suitable task. If the robot is specialized in a task that becomes less frequent in the environment, the robot has to spend more time searching for it. This search time increases the cost of specialization. A generalist, on the other hand, can accept the first task it encounters. As a result, specialists might be less efficient in environments where task types and distribution frequently change. We identify situations in which costs overcome benefits, and predict necessary changes in the system in order to avoid such disadvantages.

This paper is organized as follows. In Sec. 2] we review related works. In Sec. 3 we describe how we model leaning and specialization in our system and the task allocation strategy employed by the robots. In Sec. 4 we describe the experimental setup used for the study. In Sec. 5 we describe the experiments, and we report and discuss the results. In Sec. 6] we summarize the contributions of this work and present some directions for future research.

\section{Related Work}

Behavioral specialization has been observed in many species of animals [26]. Most works model division of labor and specialization in social insects (see [1] and [2] for an overview). These works focus almost exclusively on specialization as a means of increasing task performance by reducing external costs (e.g., travel times [15]). In these works, individuals repeatedly perform the same or a small subset of tasks, without improving in the performance of the individual tasks [6].

A way of improving task performance, other than by reducing external costs, is learning. Higher vertebrates are known to exploit this type of improvement by behavioral specialization, although it is disputed if it can be observed in social insects [5]. In robotics, improvements in task performance by learning are certainly possible (e.g., on-line adaptation of existing behaviors or learning techniques such as artificial neural networks). However, to the best of our knowledge, no works currently exist that exploit these improvements by using behavioral specialization. The general case of specialization decoupled from learning, on the other hand, has indeed been studied. Jones and Mataric studied a foraging problem, in which each individual specializes in foraging for one of two possible food types 8 . The study shows that the group behaviorally diverges depending on the ratio of food items present in the environment. Li et al. studied division of labor and specialization in an initially homogeneous swarm [10. In their work, robots behaviorally diverge by assuming different roles in a stick-pulling experiment. The study confirms that specialization usually does not occur if there are more task types than individuals available (e.g., in small groups). 
A frequently studied problem in relation to division of labor is the problem of foraging for energy. Commonly, two opposing behaviors exist (e.g., resting and foraging), that exhibit specific cost and benefits in terms of energy. The studied group has to adapt to optimize the collective energy level. Labella et al. found that, in their system, individuals effectively divide into active and passive foragers 9. Liu et al. studied a similar system with four different strategies for foraging for energy, which exhibit also an effective division of labor in the swarm [11. Recently, Ikemoto et al. proposed an adaptive mechanism for division of labor in a swarm of robots, which also divides into distinct groups that behaviorally specialize on a certain task [7].

\section{Model of Specialization}

In this section, we explain how we model the tasks and the environment, as well as the effect of learning on individual task performance. We consider an environment with different types of tasks, $\tau_{i}$, which are independent of each other. The distribution of the task types is unknown and tasks appear at random locations. The goal of the swarm is to maximize its performance, measured as the number of completed tasks in a given period of time. The nature of the tasks is such that an individual repeatedly working on a task type will become more efficient for that task type. Thus, this individual will take less time to complete tasks of this type. However, if the individual switches to another type of task, it loses part of its performance improvement for the first task type. We will refer to these phenomena as learning and forgetting, respectively.

\subsection{Learning}

We model the learning effect mentioned above as an improvement in task performance of the individual. This improvement depends on the number of successive task completions by the individual. As found in natural systems, we model the improvement as rapidly increasing in the beginning, reaching a plateau with further repetitions [5]. We define the time it takes to complete a task $\tau_{i}$ as

$$
w_{i}\left(n_{i}\right)=w_{s t d}-\frac{w_{s t d}}{k *\left(1+e^{-n_{i}+c}\right)} \quad \text { with } \quad 0<n_{i} \leq n_{\max }
$$

where $n_{i}$ is number of times a task $\tau_{i}$ was completed by the individual. $n_{i}$ is incremented each time a task $\tau_{i}$ is performed, and decremented each time a different task is performed. The standard task completion time is called $w_{s t d}$; it applies to unlearned individuals. Notice that in this work we use the same $w_{\text {std }}$ for all tasks in order to reduce the parameters of the system. $c$ is a parameter that defines after how many repetitions of a task learning starts to show a meaningful effect. The factor $k$ is used to vary the maximal time gain attainable through learning, reached after the individual successively completed $n_{\max }$ tasks of the same type. An individual reaching this state is called specialist. The resulting minimal task completion time is referred to as $w_{\text {min }}$. Fig. 1 shows a graphical representation of the learning model. 


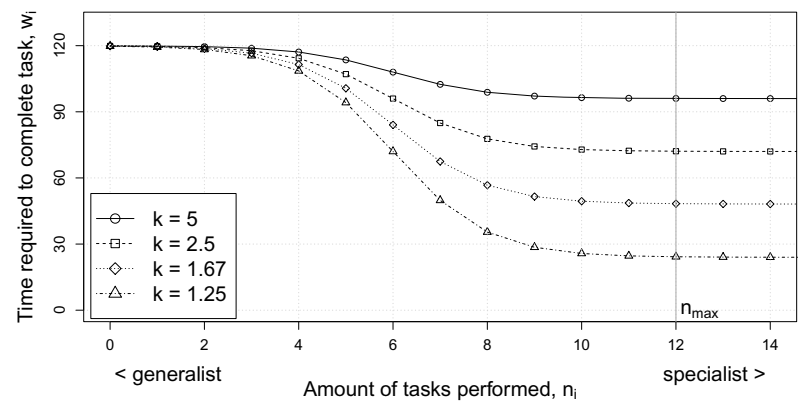

Fig. 1. The graph shows the effect of learning on the task completion time $w_{i}$ for task $\tau_{i}$. Learning applies when repeatedly working on the same type of task. Here, the standard task completion time in the unlearned state $w_{s t d}$ is $120 \mathrm{~s}$. The parameter $k$ influences the time gain of learning. The values $k=\{1.25,1.67,2.5,5\}$ shown here correspond to $20 \%, 40 \%, 60 \%$ and $80 \%$ of $w_{s t d}$ at $n_{\max }$, respectively.

\subsection{Forgetting}

In the model proposed, individuals either work on a task or search for a suitable task to work on. An individual that keeps searching gradually decreases the value of $n_{i}$, for each $i$, after traveling a distance $d_{f}$. This mechanism causes the individual to forget tasks previously completed, and thus to de-specialize while searching. Thus, individuals fall back to a generalist behavior over time. This also effectively keeps the individuals from remaining specialized on a task that is no longer available in the environment.

\subsection{Task Allocation Strategy}

The individuals of the swarm employ a simple stochastic strategy to decide if they start working on a task they encounter in the environment. The strategy is fully distributed and requires no communication between individuals, as it depends only on the individual's memory of previously completed tasks. We define the probability for an individual to start performing a task $\tau_{i}$ upon encountering it as a function of the number of completed tasks, $n_{i}$, as

$$
p_{i}\left(n_{i}\right)=\frac{1}{1+e^{-\alpha n_{i}}},
$$

with $\alpha$ being a control parameter for the steepness of the sigmoid probability curve. It influences the probability with which an individual accepts tasks of the same type: higher values of $\alpha$ will require a lower amount of tasks to be accomplished in order to reach the maximum probability. If the individual does not start to work on a task it encounters, it continues searching.

The function defined above leads to the specialization of the individual to a specific task as follows. By performing a task of type $\tau_{i}$, an individual increases its probability of repeating this task type. This causes the individual to keep on repeating tasks of type $\tau_{i}$ (if available), thus becoming a specialist for this 


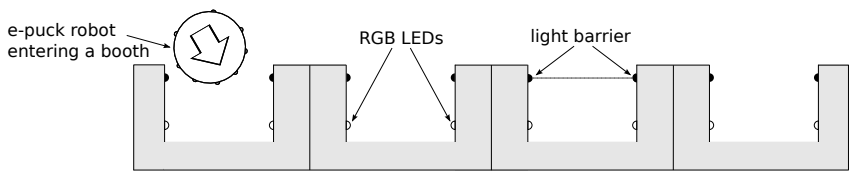

Fig. 2. Schematics of four booths arranged in an array. The light barriers detect a robot entering the corresponding booth. The color of the LEDs represent task type.

type of task. Conversely, if an individual remains inactive for a certain period of time, or performs other tasks than $\tau_{i}$, the probability of accepting tasks of type $\tau_{i}$ upon encountering them decreases due to forgetting. As the probability of accepting a task decreases for all types of task while the individual searches, individuals slowly approach a generalist behavior if they remain searching for an extended period of time. We show later that by using this mechanism, the swarm will tend to organize itself in a way that the amount of individuals specialized in each task reflects the distribution of the tasks available in the envi ronment.

\section{Experimental Setup}

In this section, we describe the experimental framework we use to test the model presented in Sec. 3. We perform our experiments in simulation, using models of the e-puck robot. The e-puck is a small wheeled robot, designed as a research and educational tool for university students [12]. In the following we describe the way we abstract the tasks in the environment, the simulation framework we use for the experiments, and the metrics we use for evaluating the system.

\subsection{Task Abstraction}

In order to overcome the limited mechanical capabilities of the e-puck, we abstract the tasks that the robots can perform with a device developed in our laboratory 3 . The device is referred to as booth. Booths can be organized into arrays, as shown in Fig. 2. Each booth features a light barrier and two RGB LEDs. The LEDs can be perceived by a robot using its color camera. The robot can navigate to the booth and enter it depending on the information perceived. The presence of a robot can be detected by the booth using its light barrier. Upon the detection of a robot, the booth reacts by changing the color of its LEDs following a user defined logic.

In our experiments, each booth represents a task that can be executed. The type of the task is encoded by the LED color of the booth. Thus, a robot can perceive which type of task a booth represents. If a robot enters a booth, it is considered to work on the corresponding task. The booth acknowledges the robot's presence by temporarily turning the LEDs to red. The robot remains inside the booth for the time $w_{i}$ required to complete the task. This time is, in general, different from robot to robot as it depends on the robot's internal state, regulated by the learning mechanisms explained in Sec. 3.3. After the robot has 

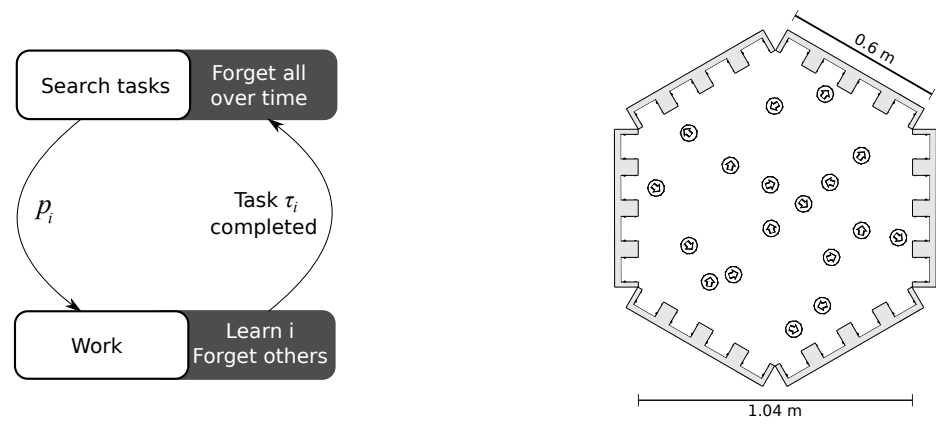

Fig. 3. Left: Finite state machine describing the behavior of the robots. Light rectangles represent actions executed by the robot, dark rectangles show the effect of learning and forgetting on the robot. A robot accepts a task it encounters with a probability $p_{i}$ (see Eq. 2). Right: Representation of the arena with e-pucks at random initial positions. Tasks can appear at each of the booths located at the boundaries of the arena, with a total of 24 tasks simultaneously available in the environment. Task types are randomly distributed.

completed its task and left, the booth stochastically selects the color to light up next. By setting the probability with which a booth selects each color, we can modify the distribution of tasks types in the environment.

\subsection{Simulation Tools}

The work presented here has been carried out using the ARGoS simulation framework [14. ARGoS is a discrete-time physics-based simulation framework developed within the Swarmanoid project 1 ARGoS can simulate various robots at different levels of detail; the experiments presented in this work are carried out in a 2-dimensional kinematics-based simulation. ARGoS simulates the whole set of sensors and actuators available on the e-puck. For our experiments we employed the wheel actuators, the color camera for tasks detection, and the IR proximity sensors for obstacle avoidance. The booth, including its sensors and actuators, is also simulated in ARGoS.

In our experiments, tasks can be of two types, $\tau_{g}$ and $\tau_{b}$, represented by green or blue LEDs, respectively. The robots can perceive tasks within a limited range using their camera, and recognize their type by their color. Fig. 3(left) shows the behavior of each robot. A robot randomly searches for a task to be performed. Upon the perception of a task (e.g., $\left.\tau_{g}\right)$, a robot has an associated probability $p_{g}$ of engaging in that task, defined by Eq. 2, Each robot is subject to the learning and forgetting mechanisms described in Sec. 3 .

The environment consists of an obstacle free hexagonal arena, with six arrays forming the walls (see Fig. 3(right)). The tasks to be performed are represented by arrays at the boundaries of the arena. Each of the arrays consists of 4 booths, for a total of 24 tasks simultaneously available in the environment. The distance

\footnotetext{
${ }^{1}$ http://www.swarmanoid.org/
} 
between an array and the one facing it on the opposite side of the arena is $1.04 \mathrm{~m}$. This dimension is such that a robot that leaves a booth cannot directly see the tasks of the booth situated diametrically.

\subsection{Specialization Measures}

To quantify the degree of specialization in the swarm, we define two measures. A measure based on the internal probability of the robots to accept a certain task type, referred to as $P$ measure, and a measure based on the frequency of task switches, referred to as $F$ measure.

The $P$ measure is based on the probability a robot has of accepting a task when encountering it. We say that a robot is specialized in a task of a certain type if its probability of accepting that type of task, as defined by Eq. 2, is greater or equal to $0.7 . P$ is the number of robots specialized according to this definition. As the $P$ measure depends on the internal probability of a robot to accept a task, it is only applicable to strategies that have such a probability.

The $F$ measure is based on the frequency of switches between different task types by a robot. It has been developed by Gautrais et al. in their study of specialization in insect colonies [6]. The individual measure $F_{i}$ consists of a value in the range $[-1,1]$, representing the degree of specialization of a robot $i$. For a sequence of $N_{i}$ tasks, it is computed as $F_{i}=1-\left(2 D_{i} / N_{i}\right)$, where $D_{i}$ is the total amount of switches between tasks of different types for robot $i$. $F$ is the average over the values of $F_{i}$ of all robots of the swarm. The value of $F$ assumes 1, 0 and -1 for a fully specialized robot, systematic switching between task types and random task allocation respectively. Different from the $P$ measure, it is independent of the underlying mechanism the robots use to select the tasks to work on.

\section{Experiments}

In this section of the paper we describe the experiments performed to study the proposed model, and present the results. We define to the ratio of tasks available in the environment as $\tau_{b} /\left(\tau_{b}+\tau_{g}\right)$, and refer to it as task ratio. It is set to 0.5 unless mentioned otherwise (equal probability of encountering one of the two types of tasks). The standard task completion time $w_{\text {std }}$ is set to $120 \mathrm{~s}$. The parameters of the learning function are set to $k=1.25$ and $c=1$, with $w_{\text {min }}=24$. This results in an $80 \%$ time gain in task completion time at the maximal learning state, reached after $n_{\max }=12$ consecutive tasks of the same type. The forgetting distance $d_{f}$ is set to $3 \mathrm{~m}$. The swarm is composed of 18 e-pucks, randomly positioned in the arena at the beginning of each experimental run. The maximum speed of the robots is set to $3 \mathrm{~cm} / \mathrm{sec}$. The task acceptance probabilities $p_{g}$ and $p_{b}$ are initialized to 0.5 ; thus, all robots start as generalist. The value of $\alpha$ has been determined by trial and error in preliminary experiments and is set to 1 . For each experimental condition we conduct 10 randomly seeded runs, for a duration of 10000 simulated seconds each. 


\subsection{Basic Properties}

In the first set of experiments we assess if the task allocation strategy presented in Sec. 3.3 successfully exploits the performance improvement available through learning. In the following, we refer to this strategy as selective strategy. We compare it to a reference strategy, the so called greedy strategy. Individuals using the greedy strategy accept, as the name indicates, any task they encounter. This allows us to quantify the performance improvement of specialization. Recall that the robots are subject to the learning and forgetting mechanisms, independently of the strategy they use for selecting their tasks. We measure the performance as the amount of tasks completed during 1000 seconds for each window of 1000 seconds after reaching the steady state $(t=5000 \mathrm{~s})$. The data is normally distributed (see supplementary on-line material [4]); we therefore report the mean $\pm \mathrm{SE}$. In case of the selective stra tegy the performance is $113.6 \pm 7.18$, and in case of the greedy strategy it is $80.8 \pm 2.51$. The performance of the two strategies is significantly different (Welch's t-test with $p=5 \%$ ).

Fig. 4 illustrates the degree at which the robots exploit learning. Two histograms show the task completion times for the greedy strategy (left) and the selective strategy (right). The histograms report data collected at the steady state $(t=5000 \mathrm{~s})$. Only results for task $\tau_{g}$ are shown; analogous results are obtained for task $\tau_{b}$. As the time spent working on a task decreases with learning, a higher frequency for low task completion times indicates an higher degree of learning. Fig. 4 (right) shows that at the steady state, when employing the selective strategy, all robots complete their task at the minimal task completion time. This indicates that maximal learning for that type of task has been reached. Fig. 4 (left) shows that tasks are performed mostly at high task completion times when using the greedy strategy. This indicates that there is poor or no learning in the swarm. As task allocatio $n$ in the greedy strategy is random, some robots manage to improve their performance temporarily by learning, and thus complete their tasks in short time. Nevertheless, as this behavior is not persistent, the results show that a strategy that exploits learning through specialization outperforms a random task allocation strategy.
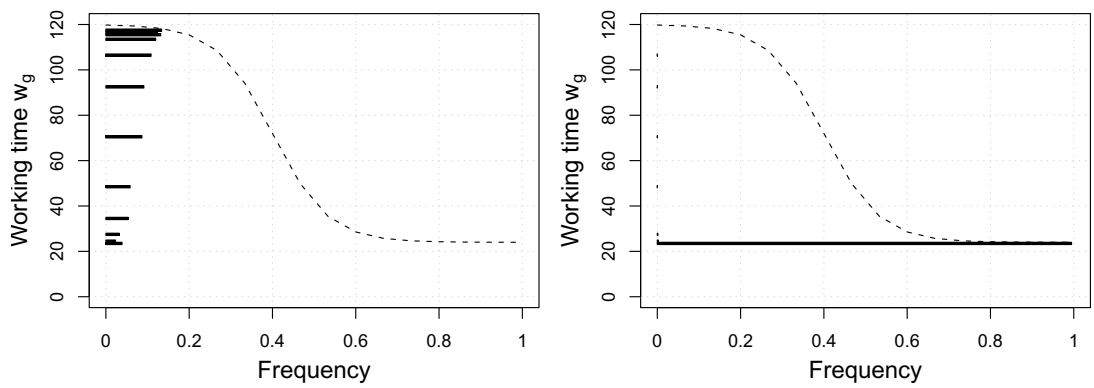

Fig. 4. Histogram for task completion time $w_{g}$, at steady state, for the greedy (left) and selective strategy (right). The learning curve (task completion time as function of the amount of tasks completed successively) is also superimposed. 

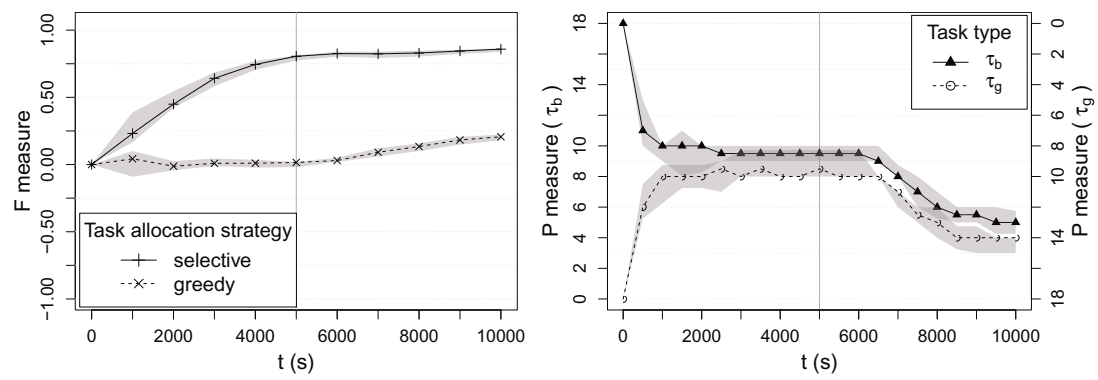

Fig. 5. Specialization of the robots of the swarm. Left: $F$ measure for both strategies; right: $P$ measure for the selective strategy only. The black line gives the median and the surrounding gray areas represents the interquartile range (IQR). The initial task ratio is 0.5 , and is changed to 0.2 at $t=5000 \mathrm{~s}$.

\subsection{Adaptivity}

In the second set of experiments we assess whether the task allocation strategy proposed is able to react to changes in the task ratio. The initial task ratio is 0.5; when the experiment reaches half its duration, the task ratio is changed to 0.2 in favor of $\tau_{g}$. We evaluate the adaptivity by using the metrics $F$ and $P$, as introduced in Section 4. For both measures we give the $25 \%, 50 \%$ and $75 \%$ quantiles, as they are not normally distributed (see on-line supplementary material 4]). Fig. [5(left) reports the specialization measure $F$ for both strategies. The $F$ measure confirms that robots using the selective strategy specialize well, as its value is very close to 1 at the steady state (just before the change in the task ratio). This indicates a high degree of specialization in the group. The plot of the selective strategy shows that after the change, the swarm stays specialized on the predominant task. Different to this, the greedy strategy does not lead to a specialization of the swarm. The growth of $F$ for the greedy strategy in the second part of the experiment reflects a higher amount of tasks of type $\tau_{g}$ in the environment.

Fig. 5)(right) visualizes what happens after the change of the task ratio. The plot reports, for the selective strategy only, the number of robots specialized in the two types of task, using the probability-based measure $P$. The graph shows that before the change of the task ratio, half the swarm is specialized for each of the two task types. This matches the task ratio. At time $t=5000 \mathrm{~s}$ the task ratio switches to 0.2 in favor of $\tau_{g}$. After a period of approximately 1000 seconds, some of the robots de-specialize from $\tau_{b}$ and subsequently specialize in $\tau_{g}$. When the steady state is reached, the number of robots specialized in $\tau_{b} / \tau_{g}$ is $4 / 14$, again matching the task ratio of the environment.

\subsection{Costs and Benefits}

In the third set of experiments we study whether specialization, which clearly has benefits in terms of task performance, entails costs that hinder the performance 

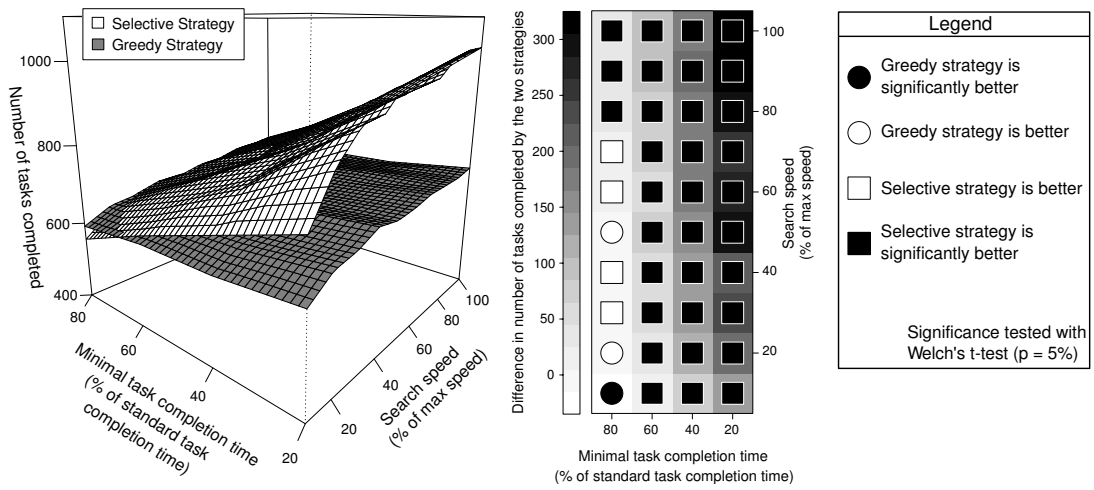

Fig. 6. Performance for different search speeds and task completion times at maximal learning. Left: observed mean of the number of completed tasks for the selective and greedy strategy (white and dark surface, respectively). SE $<2 \%$ for all values (not shown). Right: difference in number of tasks completed by the two strategies (gray shades), with indication of which strategy was better and whether the difference was statistically significant or not (see symbols in the legend).

of the swarm. The assumption is that a robot specialized for a certain task spends more time searching for it. Thus, specialists might be less efficient than generalists that accept the first task they encounter. In order to study the tradeoff between costs and benefits, we vary the costs (search time of the robots) and benefits (the minimal task completion time in the learned state). We vary the search time of the robots by changing the speed they use while searching from $10 \%$ to $100 \%$ of the maximum speed, by steps of $10 \%$. We vary the minimal task completion time $w_{\text {min }}$ from a minimum of $20 \%$ to a maximum of $80 \%$ of the standard task completion time $w_{\text {std }}$, by steps of $20 \%(k=\{5,2.5,1.67,1.25\})$.

Figure 6(left) reports the mean of the number of completed tasks performed at the end of the experiment, using the selective strategy (white surface) and the greedy strategy (dark surface), for different values of search speed and minimal task completion time. The number of completed tasks is normally distributed with a $\mathrm{SE}<2 \%$; we therefore report only the observed mean (see on-line supplementary material 4]). Fig. 6(right) shows, for all values tested, the difference in number of tasks completed by the two strategies at the end of the experiment (gray shades), and if this difference is statistically significant or not (see symbols in the legend). The plot on the left shows that the greedy strategy is less affected by the two parameters. The change in minimal task completion time has no effect as the greedy strategy does not specialize and thus does not benefit from the effects of learning. Moreover, the performance of the greedy strategy is only slightly affected by the search speed because tasks are abundant in the environment and the robots using the greedy strategy accept every task they encounter. On the other hand, the performance of the selective strategy varies considerably 
in relation to the value of the two parameters, highlighting costs and benefits of specialization. The plot on the right shows that when task completion time is $80 \%$ of the $w_{s t d}$ and the search speed $30 \%$ of the maximum speed, the greedy strategy performs better than the selective strategy. This confirms our assumption that robots specializing in a certain task are prone to loosing efficiency due to longer search times. Specialization is therefore not to be considered in terms of benefits only, as it is affected by external factors as task availability and spatial distribution, which might lower the benefits of specialization considerably.

\section{Conclusions}

In this work we focused on the topic of behavioral specialization in a swarm of robots. Behavioral specialization can often be observed in societies such as humans and social insects, as its advantages are many. Among others, it allows to exploit the improvements in task performance by learning. This means that if individuals improve their performance in a set of tasks by repeatedly carrying out those tasks, they should exploit this advantage by specializing in this set of tasks. Specialization can also entail costs: specialists may spend more time in searching for their tasks with respect to generalists.

In this work we proposed a simple model of a system in which individuals benefit from learning in terms of reduction of the time needed to perform tasks. We studied the case in which the robots can perform two types of tasks, available in the environment in a unknown ratio. We implemented a simple self-organized task allocation mechanism that leads to behavioral specialization of the individuals to one of the two tasks. We studied the system in simulation-based experiments, focusing on the response of the system to changes in the environment and on its behavior in different conditions in terms of costs and benefits of specialization. We identified cases in which costs of specialization overcome the benefits gained through learning. A task allocation strategy that does not use specialization is preferable in these cases. The results also suggest that specialization is not a good choice in highly dynamics environments, as specialists may not be able to adapt to changes fast enough. Future research will focus on the study of specialization in swarms of heterogeneous robots, where benefits and costs of specialization are linked to morphological differences between robots. Additionally, we plan to implement learning as actual improvements of behaviors instead of modeling it as an external parameter.

Acknowledgements. Marco Dorigo acknowledges support by the European Research Council through the ERC Advanced Grant "E-SWARM: Engineering Swarm Intelligence Systems" (contract 246939). Marco Dorigo, Mauro Birattari, and Arne Brutschy acknowledge support from the Belgian F.R.S.-FNRS. Marco Frison acknowledges support from "Seconda Facoltà di Ingegneria", Alma Mater Studiorum, Università di Bologna. 


\section{References}

1. Beshers, S.N., Fewell, J.H.: Models of division of labor in social insects. Annual Review of Entomology 46, 413-440 (2001)

2. Bonabeau, E., Dorigo, M., Theraulaz, G.: Swarm Intelligence: From Natural to Artificial Systems. Oxford University Press, New York (1999)

3. Brutschy, A., Pini, G., Baiboun, N., Decugnière, A., Birattari, M.: The TAM: A device for task abstraction for the e-puck robot. Tech. Rep. TR/IRIDIA/2010-015, IRIDIA, Université Libre de Bruxelles, Brussels, Belgium (2010)

4. Brutschy, A., Tran, N.L., Baiboun, N., Frison, M., Pini, G., Roli, A., Dorigo, M., Birattari, M.: Costs and benefits of behavioral specialization - Online supplementary material (2011), http://iridia.supp/IridiaSupp2011-015/

5. Dornhaus, A.: Specialization does not predict individual efficiency in an ant. PLoS Biology 6(11), e285 (2008)

6. Gautrais, J., Theraulaz, G., Deneubourg, J.-L., Anderson, C.: Emergent polyethism as a consequence of increased colony size in insect societies. Journal of Theoretical Biology 215(3), 363-373 (2002)

7. Ikemoto, Y., Miura, T., Asama, H.: Adaptive division-of-labor control algorithm for multi-robot systems. Journal of Robotics and Mechatronics 22(4), 514-525 (2010)

8. Jones, C., Matarić, M.J.: Adaptive division of labor in large-scale minimalist multirobot systems. In: 2003 IEEE/RSJ International Conference on Intelligent Robots and Systems (IROS 2003), pp. 1969-1974. IEEE Press, Piscataway (2003)

9. Labella, T.H., Dorigo, M., Deneubourg, J.-L.: Division of labour in a group of robots inspired by ants' foraging behaviour. ACM Transactions on Autonomous and Adaptive Systems 1(1), 4-25 (2006)

10. Li, L., Martinoli, A., Abu-Mostafa, Y.S.: Learning and measuring specialization in collaborative swarm systems. Adaptive Behavior 12(3-4), 199-212 (2004)

11. Liu, W., Winfield, A.F.T., Sa, J., Chen, J., Dou, L.: Towards energy optimization: Emergent task allocation in a swarm of foraging robots. Adaptive Behavior 15(3), 289-305 (2007)

12. Mondada, F., Bonani, M., Raemy, X., Pugh, J., Cianci, C., Klaptocz, A., Magnenat, S., Zufferey, J.C., Floreano, D., Martinoli, A.: The e-puck, a robot designed for education in engineering. In: Gonçalves, P.J.S., Torres, P.J.D., Alves, C.M.O. (eds.) Proceedings of the 9th Conference on Autonomous Robot Systems and Competitions, pp. 59-65. IPCB: Instituto Politècnico de Castelo Branco, Castelo Branco (2009)

13. Nitschke, G., Schut, M., Eiben, A.: Emergent specialization in biologically inspired collective behavior systems, pp. 100-140. IGI Publishing, New York (2007)

14. Pinciroli, C., Trianni, V., O'Grady, R., Pini, G., Brutschy, A., Brambilla, M., Mathews, N., Ferrante, E., Di Caro, G., Ducatelle, F., Stirling, T., Gutiérrez, A., Gambardella, L.M., Dorigo, M.: ARGoS: a pluggable, multi-physics engine simulator for heterogeneous swarm robotics. Tech. Rep. TR/IRIDIA/2011-009, IRIDIA, Université Libre de Bruxelles, Brussels, Belgium (2011)

15. Ratnieks, F.L.W., Anderson, C.: Task partitioning in insect societies. Insectes Sociaux 46(2), 95-108 (1999) 